\title{
One-Way Navier-Stokes and resolvent analysis for modeling coherent structures in a supersonic turbulent jet
}

\author{
Georgios Rigas, Oliver T. Schmidt $†$ Tim Colonius \\ California Institute of Technology, Pasadena, CA, USA \\ Guillaume A. Brès \\ CASCADE Technologies, Palo Alto, CA, USA
}

\begin{abstract}
A linear analysis of the mean flow of an isothermal ideally-expanded Mach 1.5 turbulent jet is conducted. Optimal response modes describing the fluctuating hydrodynamic and acoustic fields are obtained in a computationally efficient way by spatially marching the linearized One-Way Navier-Stokes equations. For this purpose, an adjoint-based optimization framework is proposed and demonstrated for calculating optimal boundary conditions and optimal volumetric forcing. The optimal modes are validated against modes obtained in terms of global resolvent analysis. Two scenarios are considered in the present analysis. In the first case, no restrictions are applied to the spatial forcing distribution. In the second scenario, the forcing is restricted to the nozzle plane. The resulting optimal and suboptimal modes are compared to spectral proper orthogonal modes obtained from a high-fidelity large eddy simulation. ${ }^{1}$ The implications of these observations are discussed in detail.
\end{abstract}

\section{Introduction}

Jet-noise reduction is an important goal for the commercial and military aviation communities. The use of ultrahigh-bypass-ratio turbofans on commercial aircraft has partially tackled this problem, but more stringent noise regulations require further reductions. Furthermore, the intense noise generated by highperformance military aircrafts has a significant impact on communities near airbases as well as ground crews. In this framework, the development of accurate, reduced-order models for predicting jet noise is a prerequisite for the optimization of nozzle characteristics for minimum noise.

Large-scale coherent structures, or wavepackets, are the predominant source of acoustic radiation of turbulent jets. ${ }^{2}$ Their significance in the generation of jet noise was anticipated in the early experimental work by Mollo-Christensen, and linked to an instability of the turbulent mean flow by subsequent authors. ${ }^{3,4}$ Good agreement between linear theory and experiments was found for laminar, initially laminar and forced turbulent jets. In fully turbulent unforced jets, large-scale coherent structures were also observed. A direct comparison with instability waves, however, was hindered by the intermittent behavior and broadband nature of the flow. Gudmundsson and Colonius ${ }^{5}$ were the first to achieve quantitative agreement by comparing solutions to the parabolized stability equations (PSE) with the coherent portion of the flow educed via proper orthogonal decomposition (POD) of their measurement data obtained from a microphone array in the near-field and particle image velocimetry data.

The use of global linear theory for jet flows was pioneered by Nichols and Lele ${ }^{6}$ who calculated global modes for a laminar Mach 1.5 jet. The computed eigenfunctions identified the Kelvin-Helmholtz instability of the jet shear-layer, and instability waves reminiscent of the subsonic family of modes predicted by Tam and $\mathrm{Hu} .^{7}$ The correspondence between linear global modes and coherent structures in an unforced turbulent jet was addressed by Schmidt et al. ${ }^{8}$ A remarkable agreement between the computed eigenfunctions and the

*rigas@caltech.edu

†oschmidt@caltech.edu 
wavepackets extracted from the large-eddy simulation (LES) database of a Mach 0.9 turbulent jet by Brès et al. ${ }^{9,10}$ was found. An input-output ${ }^{11-13}$ (also referred to as resolvent or frequency-response) analysis of a jet was conducted by Garnaud et al. ${ }^{14}$ for an incompressible turbulent mean flow model. The authors relate the response structure corresponding to the highest gain between optimal forcing and response to the preferred mode of the jet, i.e. the most amplified perturbation observed experientially under harmonic forcing. This preferred amplification is characterized as a pseudo-resonance with a dominant Strouhal number of 0.45. In the context of jet noise, Nichols and Jovanovic ${ }^{15}$ and Jeun et al. ${ }^{16}$ used the resolvent approach to relate optimal forcing of the momentum equations in the near-field to pressure perturbations in the far-field. Suband supersonic isothermal turbulent jets obtained as solutions to the Reynolds-avergared Navier-Stokes equations with a modified $k$ - $\epsilon$ turbulence model were investigated in both studies.

Recently a novel one-way spatial marching algorithm of slowly varying flows has been developed. ${ }^{17-19}$ The One-Way Navier-Stokes (OWNS) equations overcome the principle flaw in PSE, namely the ad hoc regularization that removes upstream propagating modes. Instead of regularizing the equations, the oneway method correctly parabolizes them based on estimating, in a computationally efficient way, the local spectrum in any cross-stream plane and designing an efficient spectral filter which eliminates modes with upstream group velocity. A full description of the methodology is provided in the references. Since the method accurately tracks the full spectrum of downstream propagating modes, the method is well suited for studying multi-modal interactions and non-modal instabilities in an efficient space-marching framework.

In the present study, we calculate linear modes using optimal OWNS equations and a resolvent analysis of the mean flow of the isothermal ideally-expanded Mach 1.5 turbulent jet calculated by Brès et al. ${ }^{1,20}$ (case B118). Results are compared to the spectral proper orthogonal modes of the LES $^{21}$ for the same flow. Finally, we address the question of whether it is sufficient to restrict the forcing in the nozzle plane or if volumetric forcing should be considered as suggested by the empirically extracted forcing distributions of Towne et al. ${ }^{22}$

\section{Methods}

\section{II.A. Resolvent Analysis}

The compressible linearized Navier-Stokes equations with additive forcing $\mathbf{f}^{\prime}$ in cylindrical coordinates and matrix form are:

$$
\frac{\partial \mathbf{q}^{\prime}}{\partial t}+\mathbf{A} \frac{\partial \mathbf{q}^{\prime}}{\partial x}+\mathbf{B}_{r} \frac{\partial \mathbf{q}^{\prime}}{\partial r}+\mathbf{B}_{\theta} \frac{\partial \mathbf{q}^{\prime}}{\partial \theta}+\mathbf{B}_{x x} \frac{\partial^{2} \mathbf{q}^{\prime}}{\partial x^{2}}+\mathbf{B}_{r r} \frac{\partial^{2} \mathbf{q}^{\prime}}{\partial r^{2}}+\mathbf{B}_{\theta \theta} \frac{\partial^{2} \mathbf{q}^{\prime}}{\partial \theta^{2}}+\mathbf{C q}^{\prime}=\mathbf{B} \mathbf{f}^{\prime}
$$

Here, $x, r, \theta$, correspond to the streamwise, radial and azimuthal directions, respectively. The equations can be written in the frequency domain, by expanding $\mathbf{q}^{\prime}(x, r, \theta, t)=\sum \hat{\mathbf{q}}_{\omega, m}(x, r) e^{-i \omega t+i m \theta}$. As the jet is periodic in the azimuthal direction, the perturbation field has been decomposed into Fourier modes with azimuthal wavenumber $m$. After spatial discretization the equations can be written in compact form as:

$$
\left(-i \omega \mathbf{I}+\mathbf{A D}_{x}+\mathbf{B}_{r} \mathbf{D}_{r}+i m \mathbf{B}_{\theta}+\mathbf{B}_{x x} \mathbf{D}_{x x}+\mathbf{B}_{r r} \mathbf{D}_{r r}-m^{2} \mathbf{B}_{\theta \theta}+\mathbf{C}\right) \hat{\mathbf{q}}:=\mathbf{L} \hat{\mathbf{q}}=\mathbf{B} \hat{\mathbf{f}},
$$

with $\mathbf{D}_{x, r}$ being the differentiation matrices in the streamwise and radial direction, and $\mathbf{L}$ the linearized Navier-Stokes operator in the frequency domain. The input-output analysis between flow response $\hat{\mathbf{q}}$ and forcing $\hat{\mathbf{f}}$ is governed by the resolvent operator $\mathcal{R}$ :

$$
\hat{\mathbf{q}}=\mathcal{R} \hat{\mathbf{f}}, \quad \mathcal{R}=\mathbf{L}^{-1} \mathbf{B} .
$$

A singular value decomposition of the discretized resolvent operator is performed to identify optimal inputoutput modes. By appropriate choice of the matrix $\mathbf{B}$, forcing can be localized in the computational domain, i.e inlet-restricted forcing.

The resolvent operator for the linearized compressible Navier-Stokes equations is spatially discretized using fourth order summation-by-parts finite differences. ${ }^{23}$ A computational domain of size $x, r \in[0,20] \times$ $[0,5]$ with $850 \times 250$ grid points clustered along $\mathrm{r}=0.5$ in the radial direction is used. An absorbing sponge zone with a width of two jet diameters is used at the inflow and outflow boundaries of the physical domain in order to prevent unwanted reflections. Far-field radiation boundary conditions are enforced at the free transverse boundary using a super-grid damping layer ${ }^{24}$ truncated by Thompson $^{25}$ characteristic conditions. The Reynolds number is fixed to $R e=U_{\infty} D / \nu=10^{4}$ for the present study. 


\section{II.B. One-Way Navier-Stokes}

The semi-discretized perturbation equations can be expressed as a spatial evolution equation:

$$
\mathbf{A} \frac{\partial \hat{\mathbf{q}}}{\partial x}=\left(i \omega \mathbf{I}-\mathbf{B}_{r} \mathbf{D}_{r}-i m \mathbf{B}_{\theta}-\mathbf{B}_{r r} \mathbf{D}_{r r}+m^{2} \mathbf{B}_{\theta \theta}-\mathbf{C}\right) \hat{\mathbf{q}}:=\mathbf{M} \hat{\mathbf{q}}
$$

Upstream propagating waves supported by equation (4) are formally removed using the one-way method, without analytically factorizing the dispersion relation, resulting in equations which are well-posed and can be solved efficiently by spatial marching. It should be emphasized that this is significantly different than other methods, i.e. PSE, which achieve a stable spatial march by numerically damping upstream propagating waves, either by using an implicit axial discretization along with a restriction on the minimum step size or by explicitly adding damping terms to the equations. The damping prevents the upstream waves from destabilizing the spatial march, but also has the unintended consequence of damping, to different degrees, all of the downstream waves.

For the derivation of the OWNS equations it is convenient to work with characteristic variables using the transformation $\boldsymbol{\phi}=\mathbf{T} \mathbf{q}$, where $\mathbf{T}$ is the diagonalizing transformation of $\mathbf{A}$, and $\boldsymbol{\phi}$ are the characteristic variables. Then, eq. (4) can be expressed as:

$$
\tilde{\mathbf{A}} \frac{\mathrm{d} \hat{\phi}}{\mathrm{d} x}=\tilde{\mathbf{M}} \hat{\boldsymbol{\phi}}
$$

The characteristics variables can be distinguished between upstream, $\phi_{+}$, and downstream, $\phi_{-}$, propagating waves. An exact parabolization requires the cancelation of the amplitude of the upstream waves, ${ }^{17}$ which results in the following differential-algebraic equation (DAE):

$$
\begin{aligned}
\tilde{\mathbf{A}}_{++} \frac{\mathrm{d} \phi_{+}}{\mathrm{d} x} & =\tilde{\mathbf{M}}_{++} \phi_{+}+\tilde{\mathbf{M}}_{+-} \phi_{-}, \\
0 & =\mathbf{U}_{-+} \phi_{+}+\mathbf{U}_{--} \phi_{-} .
\end{aligned}
$$

In the above system of equations, an eigenvalue decomposition of the matrix $\tilde{\mathbf{M}}$ has been introduced such that $\tilde{\mathbf{M}}=\mathbf{V D U}$, where the diagonal entries of $\mathbf{D}$ contain the associated eigenvalues. To circumvent the cost of the eigenvalue decomposition, a recursive one-way equation has been proposed in the references. ${ }^{17}$ In this case, the recursive OWNS read:

$$
\begin{aligned}
& \tilde{\mathbf{A}}_{++} \frac{\mathrm{d} \boldsymbol{\phi}_{+}}{\mathrm{d} x}=\tilde{\mathbf{M}}_{++} \boldsymbol{\phi}_{+}+\tilde{\mathbf{M}}_{+-} \boldsymbol{\phi}_{-}, \\
& \left(\tilde{\mathbf{M}}-i b_{+}^{j} \mathbf{I}\right) \phi^{j}=\left(\tilde{\mathbf{M}}-i b_{-}^{j} \mathbf{I}\right) \phi^{j+1}, \quad j=0, \ldots, N_{b}-1, \\
& \boldsymbol{\phi}_{-}^{N b}=0,
\end{aligned}
$$

where a set of auxiliary variables $\phi^{j}$ and a set of complex scalar parameters $b^{j}$ have been introduced. Appropriate choice of the recursion parameters parameters and order $N_{b}$ guarantee the convergence of the recursive OWNS given by (7) to the exact system (6). In both cases, the semi-discretized (discretized in $r$ ) forced one-way equations can be written in general compact form as:

$$
L \partial_{x} \phi-R \phi=\mathbf{f}, \quad \phi\left(x=x_{0}, r, \theta\right)=\phi_{0} .
$$

The above DAE can be marched efficiently in the $x$ direction with consistent initial condition $\phi_{0}$ at $x_{0}$.

In the present study, the OWNS equations are discretized in the transverse direction with the same finite-difference scheme and boundary conditions used for the global resolvent operator above. Integration of the equations in $x$ is performed using a backward differentiation formula of order 2 (BDF2). A domain of size $[0,20] \times[0,5]$ with $600 \times 250$ points is used for the solution.

\section{II.C. Optimal One-Way Navier-Stokes}

In this section we present an adjoint-based optimization framework for deriving optimal boundary conditions and optimal volumetric forcings using the OWNS equations. The first case is equivalent to the inlet-restricted 
resolvent analysis. The latter is equivalent to the unrestricted resolvent analysis with volumetric forcing. At this point the following inner products on the spatial domain and inlet-outlet boundaries are introduced:

$$
\begin{aligned}
& \langle\mathbf{a}, \mathbf{b}\rangle=\iint_{\Omega} \mathbf{a}^{H} \mathbf{b} r \mathrm{~d} x \mathrm{~d} r \\
& {[\mathbf{c}, \mathbf{d}]=\int \mathbf{c}^{H} \mathbf{d} \mathrm{d} r .}
\end{aligned}
$$

Optimization is performed by introducing the Langrange functional:

$$
\mathcal{L}\left(\phi, \phi^{*}\right)=\mathcal{J}(\phi)-\left\langle\phi^{*},\left(L \partial_{x}-R\right) \phi-f\right\rangle .
$$

Our goal is the maximization of the cost function $\mathcal{J}$, subject to the governing OWNS equations. The latter constraint is enforced by introducing the adjoint variable $\phi^{*}$.

\section{II.C.1. Optimal inlet boundary condition}

Here we present the framework for calculating the optimal boundary condition that maximizes the energy of the perturbation over the full domain without forcing. Then, the cost function can be expressed as:

$$
\mathcal{J}(\phi)=\frac{\langle\mathbf{q}, \mathbf{q}\rangle}{\left[\mathbf{q}_{0}, \mathbf{q}_{0}\right]}=\frac{\langle\phi, Q \phi\rangle}{\left[\phi_{0}, Q \phi_{0}\right]}
$$

The maximization of the objective function is achieved by finding the stationary points of the Langrange functional:

$$
\delta \mathcal{L}=\left\langle\nabla_{\phi^{*}} \mathcal{L}, \delta \phi^{*}\right\rangle+\left\langle\nabla_{\phi} \mathcal{L}, \delta \phi\right\rangle=0 .
$$

The variation of the Langrange functional with respect to the adjoint variable $\phi^{*}$ implies the solution of the direct equations. The variation with respect to the variable $\phi$ is obtained after integrating by parts the last term in (11):

$$
\left\langle\phi^{*},\left(L \partial_{x}-R\right) \phi\right\rangle=\left\langle\left(L^{*} \partial_{x}-R^{*}\right) \phi^{*}, \phi\right\rangle+\left[L^{*} \phi^{*}, \phi\right]_{x_{0}}^{x_{1}} .
$$

Subscripts 0 and 1 denote the inlet and outlet planes, respectively. Then the adjoint OWNS equations are:

$$
\left(L^{*} \partial_{x}-R^{*}\right) \phi^{*}=0, \quad \phi^{*}\left(x=x_{1}, r, \theta\right)=\phi_{1}^{*},
$$

where

$$
L^{*}=L^{H} \quad \text { and } \quad R^{*}=R^{H}+\frac{\partial L^{H}}{\partial x} .
$$

All the variations with respect to each variable have to yield zero independently, which give the following relations that have to be satisfied for the maximization of the objective function:

$$
\begin{aligned}
& \left\langle\nabla_{\phi^{*}} \mathcal{L}, \delta \phi^{*}\right\rangle=0 \quad \Rightarrow \quad L \partial_{x} \phi-R \phi=0 \\
& \left\langle\nabla_{\phi} \mathcal{L}, \delta \phi\right\rangle=0 \quad \Rightarrow \quad\left(L^{*} \partial_{x}-R^{*}\right) \phi^{*}=\frac{2 Q \phi}{\left[\phi_{0}, Q \phi_{0}\right]} \\
& \left\langle\nabla_{\phi_{0}} \mathcal{L}, \delta \phi_{0}\right\rangle=0 \Rightarrow \frac{2\left[\phi_{1}, Q \phi_{1}\right]}{\left[\phi_{0}, M \phi_{0}\right]^{2}} Q \phi_{0}=L^{H} \phi_{0}^{*} \\
& \left\langle\nabla_{\phi_{1}} \mathcal{L}, \delta \phi_{1}\right\rangle=0 \quad \Rightarrow \quad L^{H} \phi_{1}^{*}=\frac{2 Q \phi_{1}}{\left[\phi_{0}, Q \phi_{0}\right]}
\end{aligned}
$$

The above equations can be solved iteratively until the the optimal boundary condition $\phi_{0}$ is converged. The OWNS can be initialized with a random boundary condition $\phi_{0}$. The solution procedure is summarized in the following steps:

1. Solve direct equation (16a) for $\phi$ by marching downstream.

2. Initialize adjoint equation by solving (16d) for $\phi_{1}^{*}$.

3. Solve adjoint equation (16b) for $\phi^{*}$ by marching upstream.

4. Initialize direct equations by solving (16c) for $\phi_{0}$. 


\section{II.C.2. Optimal forcing}

Here we derive the framework for calculating optimal volumetric forcing that maximizes the energy of the perturbation over the full domain. In this case the cost function is expressed as:

$$
\mathcal{J}(\phi)=\frac{\langle\mathbf{q}, \mathbf{q}\rangle}{\langle\mathbf{f}, \mathbf{f}\rangle}=\frac{\langle\phi, Q \phi\rangle}{\langle\mathbf{f}, Q \mathbf{f}\rangle}
$$

The stationary points of the Langrange functional are found by setting all the variations to zero:

$$
\delta \mathcal{L}=\left\langle\nabla_{\phi^{*}} \mathcal{L}, \delta \phi^{*}\right\rangle+\left\langle\boldsymbol{\nabla}_{\boldsymbol{\phi}} \mathcal{L}, \delta \boldsymbol{\phi}\right\rangle+\left\langle\boldsymbol{\nabla}_{\mathbf{f}} \mathcal{L}, \delta \mathbf{f}\right\rangle=0 .
$$

After integrating by parts we obtain:

$$
\left\langle\phi^{*},\left(L \partial_{x}-R\right) \phi-f\right\rangle=\left\langle\left(L^{*} \partial_{x}-R^{*}\right) \phi^{*}, \phi\right\rangle+\left[L^{*} \phi^{*}, \phi\right]_{x_{0}}^{x_{1}}-\left\langle\phi^{*}, f\right\rangle .
$$

Setting again all the variations to zero, similarly to the case for the optimal boundary conditions, the following equations are obtained:

$$
\begin{array}{rll}
\left\langle\boldsymbol{\nabla}_{\boldsymbol{\phi}^{*}} \mathcal{L}, \delta \boldsymbol{\phi}^{*}\right\rangle=0 & \Rightarrow & L \partial_{x} \boldsymbol{\phi}-R \boldsymbol{\phi}=\mathbf{f} \\
\left\langle\boldsymbol{\nabla}_{\boldsymbol{\phi}} \mathcal{L}, \delta \boldsymbol{\phi}\right\rangle=0 & \Rightarrow & \left(L^{*} \partial_{x}-R^{*}\right) \boldsymbol{\phi}^{*}=\frac{2 Q \boldsymbol{\phi}}{\langle\mathbf{f}, Q \mathbf{f}\rangle} \\
\left\langle\boldsymbol{\nabla}_{\boldsymbol{\phi}_{0}} \mathcal{L}, \delta \boldsymbol{\phi}_{0}\right\rangle=0 & \Rightarrow & L^{H} \boldsymbol{\phi}_{0}^{*}=-\frac{2 Q \boldsymbol{\phi}_{0}}{\langle\mathbf{f}, Q \mathbf{f}\rangle} \\
\left\langle\boldsymbol{\nabla}_{\boldsymbol{\phi}_{1}} \mathcal{L}, \delta \boldsymbol{\phi}_{1}\right\rangle=0 & \Rightarrow & L^{H} \boldsymbol{\phi}_{1}^{*}=\frac{2 Q \boldsymbol{\phi}_{1}}{\langle\mathbf{f}, Q \mathbf{f}\rangle} \\
\left\langle\boldsymbol{\nabla}_{\mathbf{f}} \mathcal{L}, \delta \mathbf{f}\right\rangle=0 & \Rightarrow \quad \frac{\langle\boldsymbol{\phi}, Q \boldsymbol{\phi}\rangle}{\langle\mathbf{f}, Q \mathbf{f}\rangle^{2}} 2 Q \mathbf{f}=\boldsymbol{\phi}^{*}
\end{array}
$$

The equations above can be solved iteratively with termination condition the convergence of the optimal forcing. The OWNS are initialized with random forcing. The solution procedure can be summarized as follows:

1. Solve direct equation (20a) for $\phi$ by marching downstream.

2. Initialize adjoint equation by solving (20d) for $\boldsymbol{\phi}_{1}^{*}$

3. Solve adjoint equation (20b) for $\boldsymbol{\phi}^{*}$ by marching upstream.

4. Initialize direct equations by solving (20e) for $\mathbf{f}$ and (20c) for $\phi_{0}$.

\section{II.D. Spectral Proper Orthogonal Decomposition (SPOD)}

For the purpose of validating the resolvent analysis and OWNS equations, frequency-domain or spectral POD is applied to the LES data to extract coherent structures ranked on their energy content at specific frequencies $\omega$ and azimuthal wavenumbers $m$. SPOD modes are obtained from the column matrix of short-time Fourier realizations $\mathbf{Q}_{m, \omega}=\left[\hat{q}_{m, \omega}^{(1)}, \hat{q}_{m, \omega}^{(2)}, \cdots, \hat{q}_{m, \omega}^{\left(N_{b}\right)}\right]$ through the solution of the eigenvalue problem

$$
\boldsymbol{\Psi}^{*} \mathbf{Q}_{m, \omega}^{*} \mathbf{Q}_{m, \omega} \boldsymbol{\Psi}=\boldsymbol{\Lambda},
$$

where $\mathbf{Q}_{m, \omega} \mathbf{\Psi}$ is the column matrix of orthonormal Spectral POD modes whose energy is given by the eigenvalues along the diagonal of $\boldsymbol{\Lambda}$. The spectral POD modes optimally represent the data in terms of the energy norm induced by the inner product $\langle q, q\rangle$ at each given frequency. More details for the spectral POD can be found in Sinha et al. ${ }^{21}$ 
a)

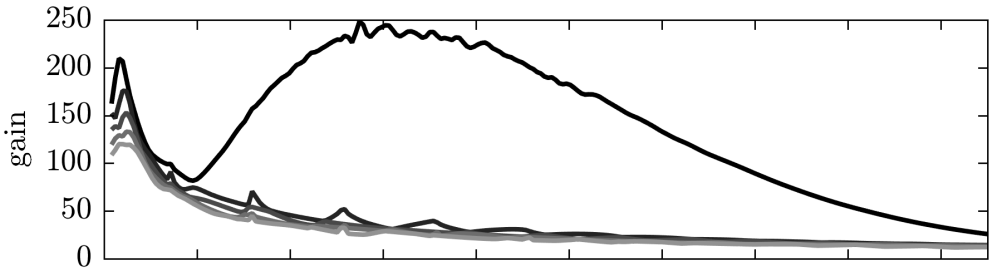

b)

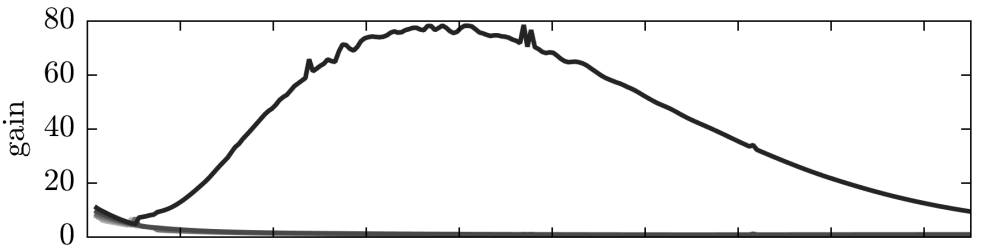

c)

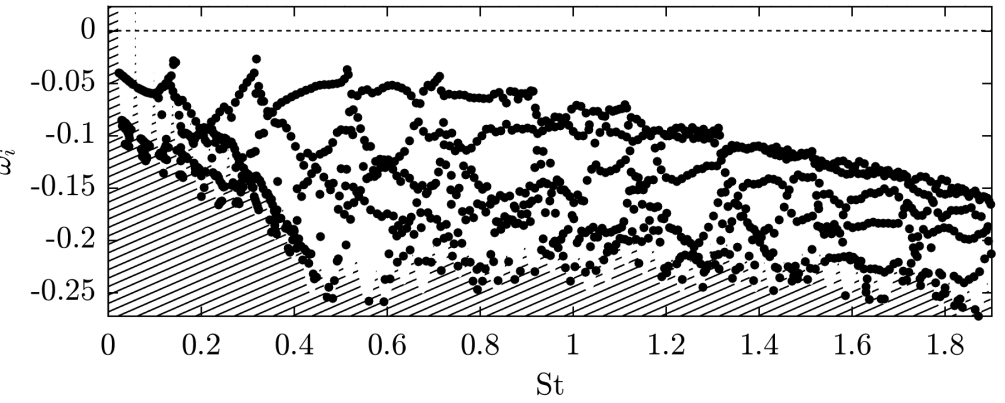

Figure 1. Comparison between resolvent gain for volumetric forcing (a), inlet plane forcing (b), and the global linear stability spectrum (c). The leading five resolvent modes where calculated for 239 frequencies. Hatched areas in the spectrum denote regions outside of the circles of convergence of the shift-and-invert Arnoldi method. 35 modes were converged at each of 48 shifts, equally spaced over the frequency domain.

\section{Results}

The five highest gain resolvent forcing and response modes were calculated for a total of 239 frequencies spanning $S t \in[0.016,1.91]$ for axisymmetric perturbations with $m=0$. Two cases were considered: first, the forcing was restricted to the inlet plane at $x=0$. This setup closely mimics the initial value problem of the PSE approach, which was used successfully in the past to model near-field wavepackets up to about the end of the potential core region for intermediate frequencies. ${ }^{21}$ Second, we consider the case where the forcing is volumetric, i.e. it is not restricted to a certain region of the flow. In the following, the resolvent modes are compared against the predictions from OWNS equations. Also, the predicted mode shapes using the above methods are compared against the SPOD modes obtained form the LES.

\section{III.A. Optimal Modes}

Figure 1 shows the comparison between inlet and volumetric forcing in terms of the gain over frequency. Additionally, the global stability spectrum of the underlying linear operator is shown for comparison. The highest gain curve for both inlet and volumetric forcing show the typical preferred amplification behavior as described by Garnaud et al. ${ }^{14}$ for their incompressible turbulent model jet. The highest gain is obtained for $S t \approx 0.6$ for volumetric, and for $S t \approx 0.8$ for inlet forcing, respectively. The Strouhal number is $S t=$ $f D / U_{\infty}$. The quantitative difference in the maximum gain is readily explained by the optimality property of input-output relation.

The global stability spectrum shown in 1c features a branch pattern related to the families of instabilities characterized by the local analysis of Tam and $\mathrm{Hu} .{ }^{7}$ These modes appear also in the resolvent gain curves. They are pronounced for the case of volumetric forcing as discrete peaks in the suboptimal modes at $S t \sim 0.18$ and integer multiples.

In figure 2, optimal pressure response modes obtained from inlet and volumetric forcing are compared to SPOD modes of the LES data for a low $(S t=0.2)$ and an intermediate $(S t=0.5)$ frequency close to the preferred amplification region. Good agreement between the resolvent response and the SPOD modes is 

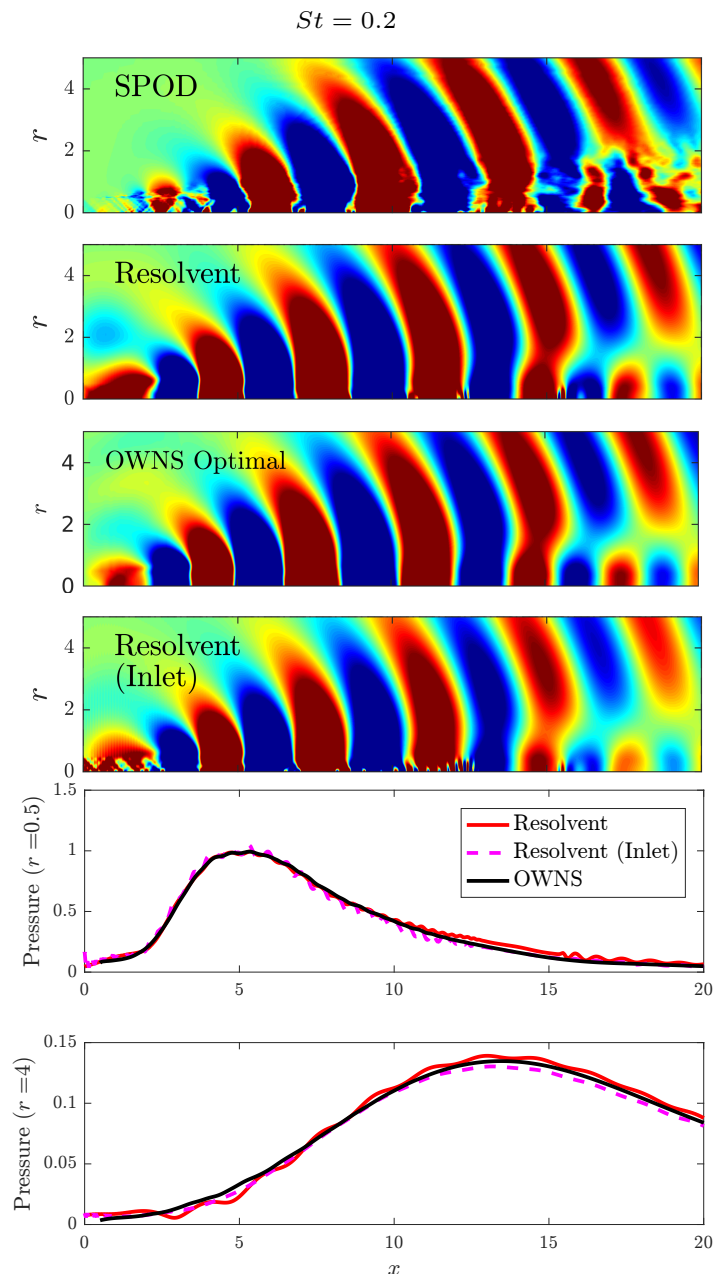
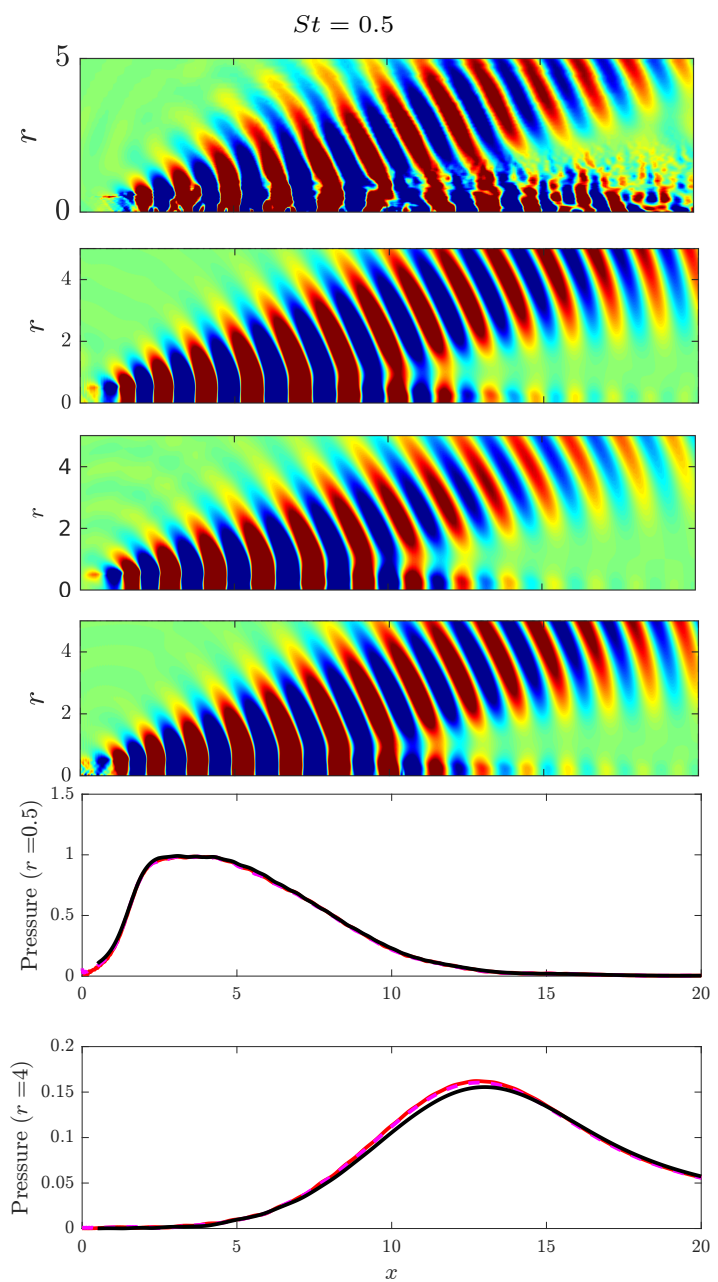

Figure 2. Comparison between the leading SPOD mode extracted from the LES data and the resolvent responses for volumetric and inlet forcing. Also, the optimal OWNS solution with volumetric forcing is shown. Pressure perturbation field for $S t=0.2$ and $S t=0.5$ with $m=0$.

observed. Details such as the superdirective acoustic radiation pattern of the wavepackets identified by the SPOD, for example, are accurately predicted by the linear analysis.

In the same figure we also present and compare the mode shapes obtained from the optimal OWNS equations with volumetric forcing against the global resolvent ones. Good agreement is obtained which validates the proposed method for obtaining optimal modes using the OWNS framework. Minor differences between the two solutions are likely to be caused due to the absorbing sponge regions in the inlet-outlet boundaries for the resolvent analysis. Sponge regions typically increase the computational cost and, since in most typical cases are inexact, they introduce error in the solution. It should be emphasized that no such absorbing regions are required for the OWNS since the methodology can be also seen as the application of an exact non-reflecting condition at each step, in order to ensure a stable spatial marching. Most importantly, the main advantage of the OWNS framework is the low computation cost which arises from the spatial marching of the equations in the slowly evolving $x$ direction instead of solving directly the global problem.

\section{III.B. Volumetric vs inlet-restricted forcing}

In figure 2 the perturbation pressure field with inlet-restricted forcing is also plotted for the two frequencies, showing good agreement with the volumetric forcing case. The amplitude of the pressure perturbation is compared at the bottom figures. The quantitative comparison of the pressure near field at $r=0.5$ and $r=4$ at the bottom graph of the figure shows almost identical mode shapes for the methods used. For comparison, each two-dimensional pressure field has been normalized with its maximum value. These two locations were chosen as representative regions where hydrodynamic instabilities and acoustic radiation 

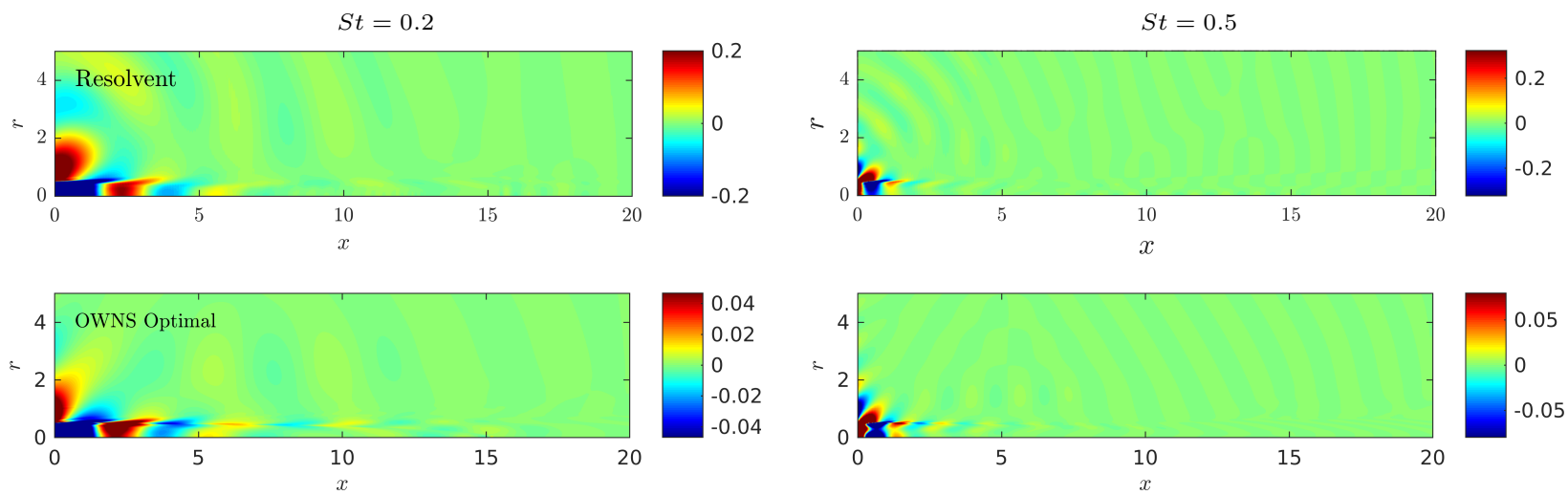

Figure 3. Optimal volumetric forcing obtained from resolvent analysis and OWNS equations. Pressure perturbation field (real part) for $S t=0.2$ and $S t=0.5$ with $m=0$.
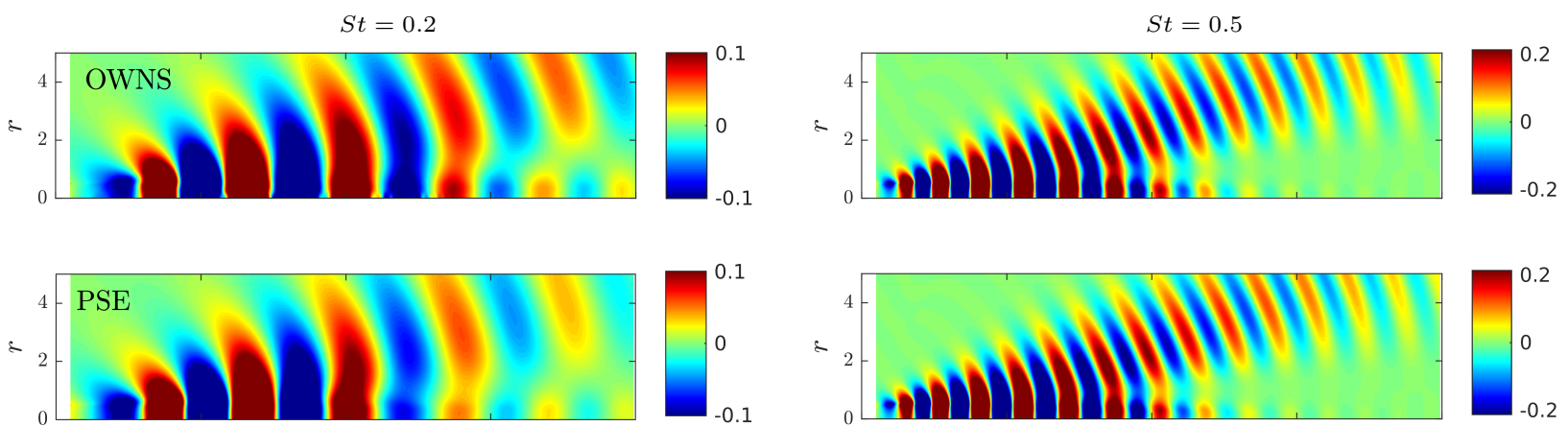

Figure 4. OWNS and PSE solutions. The same amplitude initial condition was supplied to both PSE and OWE to initialize the spatial marching, corresponding to the local eigenvector of the Kelvin-Helmholtz mode at the nozzle exit. Acoustic radiation is damped in the PSE results when compared to the OWE predictions.

dominate, respectively.

Volumetric and inlet-restricted (boundary) forcing produce the same modal shape for the perturbation field. However, the resolvent gain compared to the inlet restricted resolvent is approximately 3 times higher, implying that boundary forcing is not the optimal excitation for the flow. Optimal excitation is achieved by volumetric forcing which is localized close to the nozzle. The optimal volumetric forcing distributions are shown in figure 3, as predicted from resolvent and OWNS. The forcing shapes suggest that, at least for intermediate frequencies, it is sufficient to regard the wavepackets as purely convective instability waves which are generated close to the inlet and subsequently undergo spatial exponential growth while being convected downstream. This observations also explains the success of space-marching models in the corresponding frequency regime, for which the Kelvin-Helmholtz eigenvector is typically used as initial condition.

Finally, the local Kelvin-Helmholtz instability close to the nozzle approximates to a reasonable extend the optimal boundary condition. In this case, the response corresponding to the optimal resolvent forcing can be interpreted as the optimal boundary condition for the spatial marching problem (i.e PSE or OWNS). We show this by marching the OWNS and PSE equations with initial condition the local spatially unstable Kelvin-Helmholtz eigenvector close to the nozzle exit at $x=0.5$. Results are shown in figure 4. Both methods were initialized with the same amplitude Kelvin-Helmhotz eigenvector at $x=0.5$. As in the case of subsonic jets, ${ }^{17,18}$ PSE significantly damp the acoustic waves. However, this effect is less pronounced for supersonic jets compared to subsonic ones. The PSE acoustic field reaches up to $50 \%$ acoustic attenuation at the end of the computational domain for $S t=0.2$, when compared to the OWNS solution.

\section{III.C. Suboptimal modes}

As shown above, for a wide range of frequencies dominated by the shear-layer Kelvin-Helmholtz instability, $S t \in[0.2,1.8]$, the local spatially unstable eigenmode close to the nozzle exit is a good candidate for optimally exciting the flow. This is also evident in the gain curves presented in figure 1, where the optimal mode is clearly separated in terms of amplification from the suboptimal ones. However, it is observed that at low 

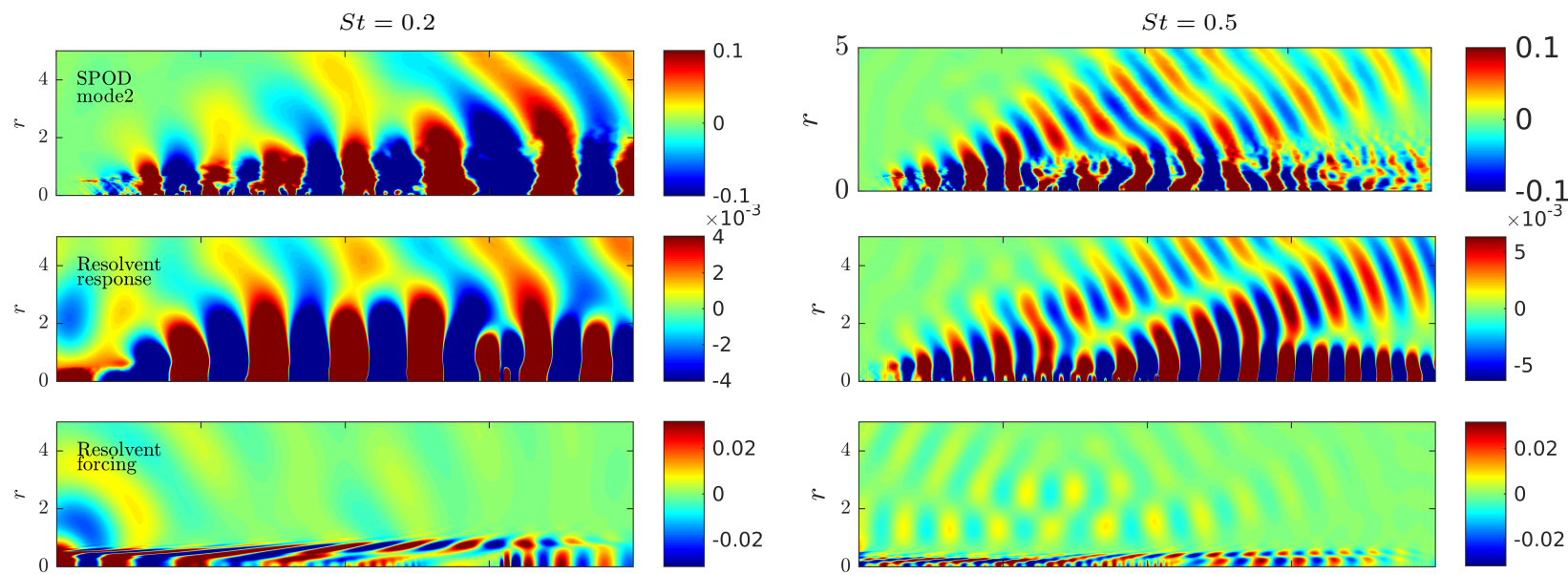

Figure 5. Second most energetic SPOD mode obtained from the LES compared against first suboptimal (second most amplified) resolvent mode. The resolvent forcing is also shown. Pressure perturbation field (real part) for $S t=0.2$ and $S t=0.5$ with $m=0$.

frequencies this mode is not the dominant one. The previously suboptimal modes at higher frequencies increase in gain towards lower frequencies and, in combination with the attenuation of the Kelvin-Helmholtz instability, they become the dominant mechanism dictating the optimal flow response. The gain curves of the five leading resolvent modes almost collapse at very low frequencies. This suggests that the flow response cannot be modeled in terms of just one dominant response structure at this frequency. The significance of the suboptimal modes was also highlighted by Jeun et al. ${ }^{16}$ for the acoustic far-field of the same jet. ${ }^{1}$ The above observations have significant implications for the modeling of the flow at low frequencies. In the same time, they explain the failure of space-marching techniques to capture the flow patterns at these range of frequencies,${ }^{5,21}$ since they are typically initialized with the Kelvin-Helmholtz eigenmode close to the nozzle exit.

The second most energetic spectral POD mode is shown in figure 5 and compared against the first suboptimal resolvent mode (second most amplified), for the two frequencies examined previously. For the lower frequency, $S t=0.2$, the computational domain does not suffice for an accurate comparison of the acoustic fields due to the wavelength decrease of the perturbations. Furthermore, the comparison becomes more challenging at low frequencies from a data processing aspect due to the increased number of data realizations required to converge the SPOD modes. However, it appears that the hydrodynamic field shares qualitatively similar features between the POD and resolvent modes. For $S t=0.5$ a double superdirective acoustic beam is observed in both the SPOD and resolvent response, showing good agreement. This is directly related to the two coherent hydrodynamic wavepackets which emerge before and after the end of the potential core of the jet. Also, the associated volumetric forcing is shown in the same figure, which reveals a qualitatively different behavior than that of the optimal modes discussed above. The suboptimal modes have a spatially extended optimal forcing distribution, compared to the optimal ones for which the forcing is localized close to the nozzle. In accordance with their response, also two distinct volumetric forcing mechanisms exist, which force and give rise to each wavepacket independently. The qualitative similarity of the forcing pattern, allows us to expect similar behavior of these suboptimal modes at very low frequencies. Finally, regarding the inlet-restricted optimal forcing, it follows naturally that the amplification of the suboptimal modes is much smaller, since the volumetric forcing is a necessary mechanism for maximum amplitude growth.

\section{Conclusions}

Optimal OWNS equations have been proposed to examine the coherent structures of an isothermal ideallyexpanded Mach 1.5 turbulent jet. The framework is general and different cost functions can be introduced for the optimization procedure. Here, we have examined optimal volumetric forcing and optimal inlet boundary conditions that maximize the energy of the fluctuating field. It has been shown that this is equivalent to a global resolvent analysis with volumetric forcing and inlet-restricted forcing. Important advantage of the OWNS framework is the low computation cost which arises from the spatial marching of the equations in 
the slowly evolving $x$ direction. This characteristic makes the method promising for examining the stability and coherent structures of fully three-dimensional and inhomogeneous slowly varying flows, such as jets and boundary layers.

For the supersonic jet examined here, we showed that volumetric and inlet-restricted forcing produce the same optimal modal shape for the perturbation field in the frequency range dominated by the spatially unstable Kelvin-Helmholtz mechanism. However, higher amplification gains can be obtained with volumetric forcing. For this regime, the optimal forcing is localized in a region close to the nozzle exit and the local Kelvin-Helmholtz mode approximates to a reasonable extend the optimal boundary condition. Similarly to the PSE predictions, the optimal response modes are in good agreement with the frequency POD modes extracted from the LES data for both the hydrodynamic and acoustic fields. Good agreement is obtained also for suboptimal resolvent modes when compared against the second most energetic POD modes. Although the suboptimal modes for the frequency range $0.2<S t<1.8$ have a less pronounced role in terms of amplification, for very low frequencies, approximately $S t<0.15$, they dominate the flow response. This explains the failure of PSE models to capture the flow patterns at low frequencies, since they are typically initialized with the Kelvin-Helmholtz eigenmode. Our findings suggest that, instead of a single spatially unstable mode, a family of modes are responsible for the flow pattern at these frequencies. These modes can be modelled using resolvent analysis and optimal OWNS, since they provide an optimal framework for capturing modal and non-modal instabilities.

\section{Acknowledgments}

This research was supported in part by a grant from the Office of Naval Research (grant No. N00014-16-1$2445)$ with Dr. Knox Millsaps as program manager. The LES study was performed at Cascade Technologies, with support from ONR and NAVAIR SBIR project, under the supervision of Dr. John T. Spyropoulos. The main LES calculations were carried out on DoD HPC systems in ERDC DSRC. O.S. was also supported in part by DFG grant No. SCHM 3114/1-1. G.R. and T.C. also acknowledge the support of the Boeing Company through a Strategic Research and Development Relationship Agreement CT-BA-GTA-1.

\section{References}

\footnotetext{
${ }^{1}$ Brès, G., Nichols, J., Lele, S., and Ham, F., "Towards best practices for jet noise predictions with unstructured large eddy simulations," AIAA paper 2012-2965, 2012.

${ }^{2}$ Jordan, P. and Colonius, T., "Wave packets and turbulent jet noise," Annual Review of Fluid Mechanics, Vol. 45, 2013, pp. 173-195.

${ }^{3}$ Michalke, A., "Instability of a compressible circular free jet with consideration of the influence of the jet boundary layer thickness," Z. für Flugwissenschaften, Vol. 19, No. 8, 1971, pp. 319-328.

${ }^{4}$ Crighton, D. G. and Gaster, M., "Stability of slowly diverging jet flow," Journal of Fluid Mechanics, Vol. 77, No. 02, 1976, pp. 397-413.

${ }^{5}$ Gudmundsson, K. and Colonius, T., "Instability wave models for the near-field fluctuations of turbulent jets," Journal of Fluid Mechanics, Vol. 689, 2011, pp. 97-128.

${ }^{6}$ Nichols, J. W. and Lele, S. K., "Global modes and transient response of a cold supersonic jet," Journal of Fluid Mechanics, Vol. 669, 2011, pp. 225-241.

${ }^{7}$ Tam, C. K. W. and Hu, F. Q., "On the three families of instability waves of high-speed jets," Journal of Fluid Mechanics, Vol. 201, 1989, pp. 447-483.

${ }^{8}$ Schmidt, O. T., Towne, A., Colonius, T., Jordan, P., Jaunet, V., Cavalieri, A. V. G., and Brès, G. A., "Super-and multi-directive acoustic radiation by linear global modes of a turbulent jet," AIAA paper 2016-2808, 2016.

${ }^{9}$ Brès, G. A., Jordan, P., Colonius, T., Le Rallic, M., Jaunet, V., and Lele, S. K., "Large eddy simulation of a Mach 0.9 turbulent jet," Proceedings of the Summer Program, Center for Turbulence Research, Stanford University, 2014.

${ }^{10}$ Brès, G. A., Jaunet, V., Le Rallic, M., Jordan, P., Colonius, T., and Lele, S. K., "Large eddy simulation for jet noise: the importance of getting the boundary layer right," AIAA paper 2015-2535, 2015.

${ }^{11}$ Jovanović, M. R. and Bamieh, B., "Componentwise energy amplification in channel flows," Journal of Fluid Mechanics, Vol. 534, 2005, pp. 145-183.

${ }^{12}$ McKeon, B. J. and Sharma, A. S., "A critical-layer framework for turbulent pipe flow," Journal of Fluid Mechanics, Vol. 658, 2010, pp. 336-382.

${ }^{13}$ Beneddine, S., Sipp, D., Arnault, A., Dandois, J., and Lesshafft, L., "Conditions for validity of mean flow stability analysis," Journal of Fluid Mechanics, Vol. 798, 2016, pp. 485-504.

${ }^{14}$ Garnaud, X., Lesshafft, L., Schmid, P. J., and Huerre, P., "The preferred mode of incompressible jets: linear frequency response analysis," Journal of Fluid Mechanics, Vol. 716, 2013, pp. 189-202.

${ }^{15}$ Nichols, J. W. and Jovanovic, M. R., "Input-output analysis of high-speed jet noise," Proceedings of the Summer Program, Center for Turbulence Research, Stanford University, 2014.
} 
${ }^{16}$ Jeun, J., Nichols, J. W., and Jovanović, M. R., "Input-output analysis of high-speed axisymmetric isothermal jet noise," Physics of Fluids, Vol. 28, No. 4, 2016, pp. 047101.

${ }^{17}$ Towne, A. and Colonius, T., "One-way spatial integration of hyperbolic equations," Journal of Computational Physics, Vol. 300, 2015, pp. 844-861.

${ }^{18}$ Towne, A. and Colonius, T., "Improved parabolization of the Euler equations," AIAA paper 2013-2171, 2013.

${ }^{19}$ Rigas, G., Colonius, T., and Beyar, M., "Stability of wall-bounded fows using one-way spatial integration of Navier-Stokes equations," AIAA paper 2017-1881, 2017.

${ }^{20}$ Brès, G. A., Ham, F. E., Nichols, J. W., and Lele, S. K., "Unstructured Large Eddy Simulations of Supersonic Jets," AIAA, Vol. 55, No. 4, 2017, pp. 1164-1184.

${ }^{21}$ Sinha, A., Rodríguez, D., Brès, G. A., and Colonius, T., "Wavepacket models for supersonic jet noise," Journal of Fluid Mechanics, Vol. 742, 2014, pp. 71-95.

${ }^{22}$ Towne, A., Colonius, T., Jordan, P., Cavalieri, A., and Brès, G. A., "Stochastic and nonlinear forcing of wavepackets in a Mach 0.9 jet," AIAA paper 2015-2217, 2015.

${ }^{23}$ Mattsson, K. and Nordström, J., "Summation by parts operators for finite difference approximations of second derivatives," Journal of Computational Physics, Vol. 199, No. 2, 2004, pp. 503-540.

${ }^{24}$ Appelö, D. and Colonius, T., "A high-order super-grid-scale absorbing layer and its application to linear hyperbolic systems," Journal of Computational Physics, Vol. 228, No. 11, 2009, pp. 4200-4217.

${ }^{25}$ Thompson, K. W., "Time dependent boundary conditions for hyperbolic systems," Journal of Computational Physics, Vol. 68, No. 1, 1987, pp. 1-24.

11 of 11 